\section{NOVEL GLYCOMIMETICS INHIBIT GLY-LDL INDUCED SMOOTH MUSCLE CELL CALCIFICATION VIA CREB}

Gary P Sidgwick*, Ria Weston, Alan M Jones, Fiona L Wilkinson, Yvonne Alexander Manchester Metropolitan University

10.1136/heartjnl-2017-311726.181

Advanced glycated end-products (AGEs) are known drivers of cardiovascular complications such as vascular calcification, which is currently untreatable, and are increased in diabetic subjects in part due to oxidative stress and poor glycaemic control. Our previous studies using smooth muscle cells (SMCs) isolated from patients with peripheral arterial disease (PAD) have implicated a number of signalling pathways involved in their osteogenic differentiation in vitro, including OPG/RANK. We have also shown that alterations in the cell surface glycocalyx regulates cell function, suggesting that nonsugar glycosaminoglycan mimics can potentially modulate cell phenotypes.

We aim to investigate how modified LDL and PAD serum affects the progression of calcification of SMCs in vitro and whether this pathology can be prevented by novel glycosaminoglycan mimics, using qPCR, ELISA, Alkaline phosphatase (ALP) activity, and Western blotting.

Gly-LDL $(10 \mu \mathrm{g} / \mathrm{ml})$ increased an early marker of calcification (ALP activity) at 4 days and enhanced calcification at 21 days, compared to controls $(\mathrm{p}<0.05)$ as shown by alizarin red staining. PAD serum accelerated calcification, which was apparent after 10 days $(\mathrm{p}<0.05)$. The glycomimetics significantly inhibited both gly-LDL and PAD serum-induced mineralisation, and reduced gly-LDL induced ALP activity at day 4 $(p<0.05)$. In the gly-LDL-treated SMCs, secreted levels of osteocalcin (OCN), a promoter of osteogenic differentiation, were reduced when treated with the glycomimetics, whereas osteopontin (OPN) and osteoprotegerin (OPG), inhibitors of calcification, were increased compared to gly-LDL alone. A similar trend was observed in PAD serum-treated SMCs, with glycomimetics reducing OCN and increasing OPN secretion. A phospho-kinase array analysis of gly-LDL-treated SMCs was performed to identify underlying mechanism of action. GlyLDL increased phosphorylation of cyclic AMP response element-binding protein (CREB), TOR, and the SRC proteins LYN, YES and CHK-2 compared with untreated control, which was attenuated with the addition of the glycomimetics. A number of upstream activators of CREB were targeted using known pharmacological inhibitors in SMCs treated with glyLDL. The MEK inhibitor U0126 accelerated calcification, increasing both ALP activity and expression of receptor for AGEs (RAGE), a key receptor implicated in vascular calcification, compared to gly-LDL alone, suggesting that MEK may be a mediator of mineralisation process via phosphorylation of CREB.

Glycomimetics have potential as an anti-calcification strategy, inhibiting mineralisation in SMCs induced by both glyLDL and patient serum in vitro. The protective effect of glycomimetics against calcification may occur via regulation of CREB phosphorylation and subsequent modulation of downstream osteogenic markers, including upregulation of OPN and OPG and reduction of OCN, leading to the development of therapeutics to treat vascular calcification.

\section{UROTENSIN II INDUCES CARDIOMYOCYTES HYPERTROPHY VIA ACTIVATION OF THE MAPK AND CAMKII SIGNALLING PATHWAYS}

Hadeel Al Ali* ${ }^{*}$ David Lambert, Glenn Rodrigo. University of Leicester

\subsection{6/heartjnl-2017-311726.182}

Introduction The normal concentration of circulating Urotensin II (UII) is elevated in heart failure ( $\mathrm{Ng}$ et al. 2002). UII has been shown to be involved the development of pathological cardiac hypertrophy (Tzanidis et al. 2003). The aim of this study is to investigate the cellular mechanism by which UII results in ventricular hypertrophy.

Methodology Adult Rat Ventricular Myocytes (ARVMs) were isolated from male Wistar rats by enzymatic digestion and placed into primary culture. ARVMs were cultured in six wells plate for up 48 hours. To induce hypertrophy, cells were treated with UII $(200 \mathrm{nM})$ or phenylephrine $(10 \mu \mathrm{M})$, and hypertrophy quantified as Length/Width (L/W) ratio from photomicrographs. Results Cultured AVRMs exposed to either phenylephrine or UII developed hypertrophy in a timedependent manner, with a significant reduction in $\mathrm{L} / \mathrm{W}$ ratio after incubation with UII for 24 hours in comparison with control group $(4.25 \pm 0.06, \mathrm{n}=362$ vs. $4.45 \pm 0.06, \mathrm{n}=335)$ $(\mathrm{p}<0.05)$ or 48 hours $(3.99 \pm 0.06, \mathrm{n}=209$ vs. $4.53 \pm 0.10$, $n=126)(p<0.0001)$. Treatment of AVRMs with phenylephrine induced a similar level of hypertrophy to that seen by UII, to alteration in the morphology of myocytes, there was a significant reduction in $\mathrm{L} / \mathrm{W}$ ratio after incubation with phenylephrine for 24 hours in comparison with control group (4.08 $\pm 0.05, \mathrm{n}=338$ vs. $4.45 \pm 0.06, \mathrm{n}=335)(\mathrm{p}<0.05)$ or 48 hours $(3.77 \pm 0.08, \mathrm{n}=141$ vs. $4.53 \pm 0.10, \mathrm{n}=126) \quad(\mathrm{p}<0.0001)$. To study the role of ERK1/2, P38 and CaMKII signalling in the UII-induced hypertrophy, cultured ARVMs were treated with either $5 \mu \mathrm{M}$ of ERK1/2 inhibitor (PD 184352), $10 \mu \mathrm{M}$ of P38 inhibitor (SB 202190) or $5 \mu \mathrm{M}$ CaMKII (KN-93) for $30 \mathrm{~min}$ prior to stimulation with UII $(200 \mathrm{nM})$. Inhibition of all three signalling pathways completely blocked the UII-induced hypertrophy after 48 hours; from $(3.87 \pm 0.05, \mathrm{n}=420)$ in UII group to $(4.64 \pm 0.08, \mathrm{n}=195)$ (UII $+\mathrm{PD}) \quad(\mathrm{p}<0.0001)$ and from $(3.87 \pm 0.05, \mathrm{n}=420)$ in UII group to $(4.86 \pm 0.09, \mathrm{n}=212)$ (UII $+\mathrm{SB})(\mathrm{p}<0.0001)$. Inhibition of CaMKII from $(3.39$ $\pm 0.04, \mathrm{n}=386)$ in UII group to $(4.14 \pm 0.06, \mathrm{n}=223)$ (UII + CaMKII) $(\mathrm{p}<0.0001)$.

Conclusion These data show that the ERK1/2, p38 and CaMKII signalling pathways are involved in the hypertrophic response to UII. I am currently using Western Blot to confirm the involvement of ERK1/2, P38 and CaMKII pathways.

\section{REFERENCES}

1 Ng LL, Loke I, O'Brien RJ, Squire IB, Davies JE. Circulation 2001;106(23):28772880

2 Tzanidis A, Hannan RD, Thomas WG, Onan D, Autelitano DJ, See F, Kelly DJ, Gilbert RE, Krum H. Circulation Research: Journal of the American Heart Association, 203:93(3):246-253

\section{MISALIGNMENT OF TRANSCATHETER AORTIC VALVE IMPLANTS WITH THEIR DESTINATION AORTIC ROOT RESULTS IN DIMINISHED VALVE PERFORMANCE}

Jacob Salmon*. University College London

10.1136/heartjnl-2017-311726.183 

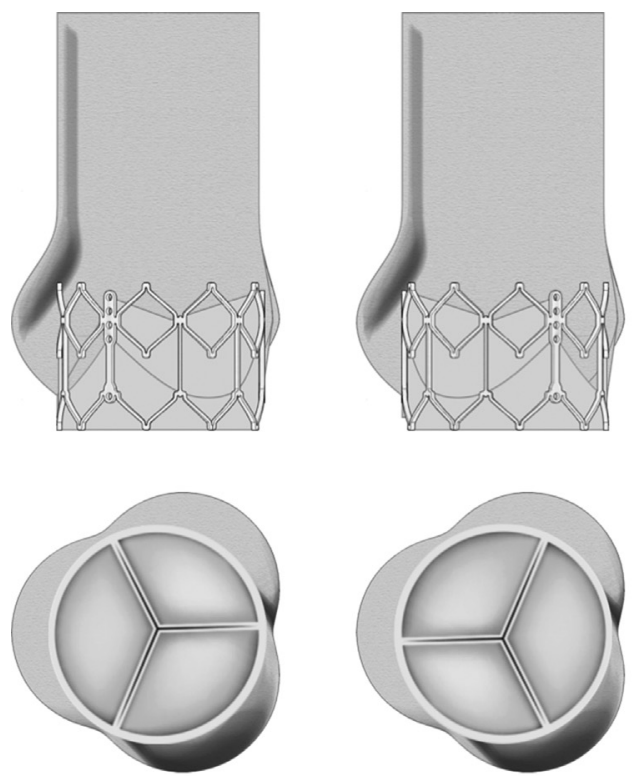

Commissure on commissure, no native leaflets

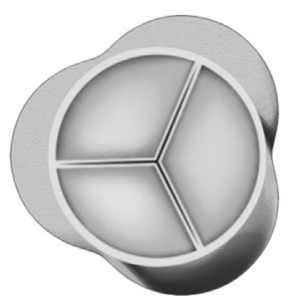

Commissure on belly, no native leafiets
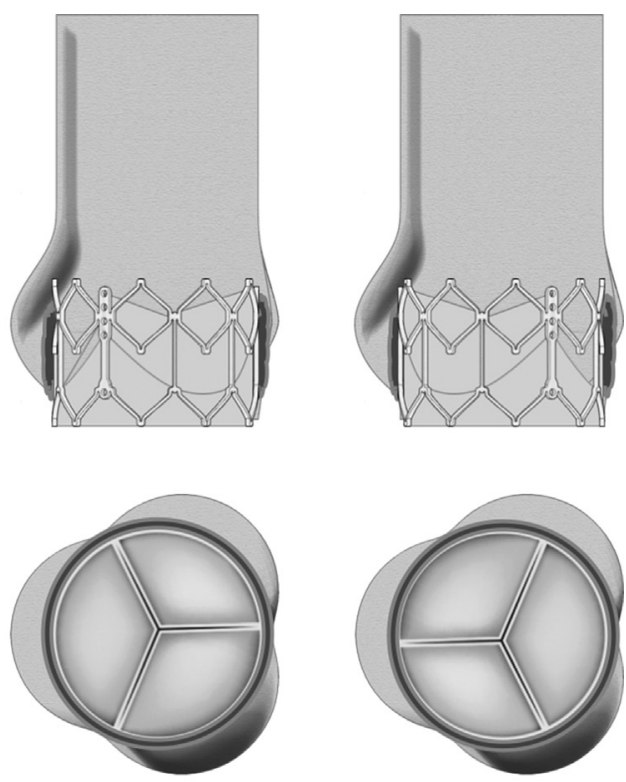

Commissure on commissure, with native leaflets

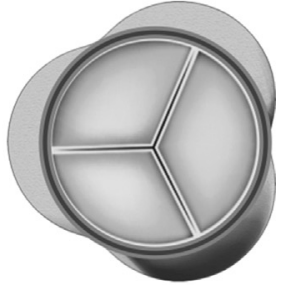

Commissure on belly, with native leaflets

\section{Abstract 185 Figure 1}

Purpose Many Transcatheter Aortic Valve Implant (TAVI) devices currently on the market offer little control over the rotational alignment of the bioprosthesis with the aortic root they are being inserted into, and the resultant flow dynamics are not fully understood. This study aims to identify how the valve orientation affects haemodynamics, both neglecting and including the presence of the native leaflets.

Methods Using an in vitro approach, commercially available TAVI devices were implanted into a mock silicone aortic root in four configurations encompassing the variations of aligned/ non-aligned and without/with native leaflets present (Figure 1). Global flow information was obtained using pressure and flow transducers, and 2D Particle Image Velocimetry (PIV) was used to provide local and instantaneous flow data across the various configurations.

Results Analysis of the PIV images shows that a vortex is generated above each opening leaflet of the TAVI device, and another above or within the sinus of the root. If the commissures of the root and prosthetic valve are aligned, this results in these vortices merging to form only one major vortex. On the contrary, if the valve and root are misaligned, the two vortices remain separate, each impinging the central jet of fluid flow (Figure 2). This raises pressure losses, decreasing valve performance, and increasing turbulence and Reynolds shear stresses. This effect occurs both with and without the native leaflets, whose presence results in a significant reduction in the flow velocity observed in the sinuses.

Conclusion These results show that misalignment of a TAVI device with its destination root can lead to an alteration in vortical behaviour, leading to diminished valve performance. There is also increased occurrence of conditions which are conducive to the generation of thrombosis. The presence of native leaflets does not mitigate this vortical behaviour, and also reduces the systolic flow in the sinuses, possibly leading to increased stagnation. This fluid behaviour should be taken into account when considering design and insertion of TAVI devices.
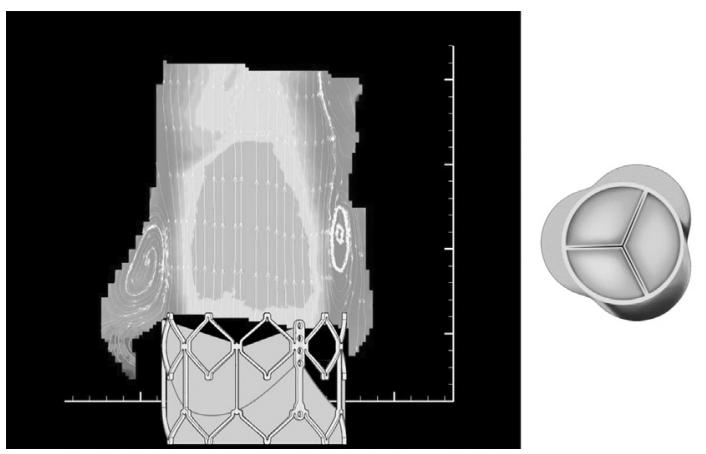

Abstract 185 Figure 2

\section{REDUCED PERICYTE INSULIN SIGNALLING CAUSES ABNORMAL DEVELOPMENTAL ANGIOGENESIS}

${ }^{1}$ Nele Warmke*, ${ }^{1}$ Kathryn J Griffin, ${ }^{2}$ Charles I Slater, ${ }^{1}$ Andrew MN Walker, ${ }^{1}$ Nadira Y Yuldasheva, ${ }^{1}$ Richard M Cubbon. ' University of Leeds; ${ }^{2}$ University of Cambridge

\subsection{6/heartjnl-2017-311726.184}

Introduction Microvascular endothelial cells (ECs) are surrounded and supported by mural cells called pericytes. Via paracrine signalling and gap junctions, pericytes interact with ECs, modulating angiogenesis and vessel homeostasis. Diabetes-associated vascular complications are characterised by vascular leakage and dysfunctional neovascularization, both associated with pericyte loss in various vascular beds, including the retina. Pericytes are particularly sensitive to a hyperglycaemic environment, which results in their apoptosis and subsequent changes in vascular health. Insulin also has important 